\title{
An improvement of an estimate for finite additive bases
}

by

\section{G. HoRváth (Dunaújváros)}

1. Introduction. Let $\mathcal{A}=\left\{a_{1}, \ldots, a_{k}\right\}$ be a set of integers such that $0 \leq a_{1}<\cdots<a_{k}$, and let $\mathcal{A}+\mathcal{A}=\left\{a_{l}+a_{m} \mid a_{l} \in \mathcal{A}, a_{m} \in \mathcal{A}\right\}$. If $n$ is a natural number and $\{0,1, \ldots, n\} \subseteq \mathcal{A}+\mathcal{A}$ then $\mathcal{A}$ is called a 2-basis. Let $k=k(n)$ be the smallest integer for which a 2-basis for $n$ with $k$ elements exists, and let $\mathcal{A}$ be such a minimal 2-basis.

Since $n+1 \leq|\mathcal{A}+\mathcal{A}| \leq\left(\begin{array}{c}k \\ 2\end{array}\right)+k=\left(k^{2}+k\right) / 2$, we have

$$
\limsup _{n \rightarrow \infty} \frac{n}{k^{2}} \leq \frac{1}{2} \text {. }
$$

On the other hand, it is not hard to see that the set

$$
\{0,1,2, \ldots,[\sqrt{n}-1],[\sqrt{n}], 2[\sqrt{n}], 3[\sqrt{n}], \ldots,[\sqrt{n}+1] \cdot[\sqrt{n}]\}
$$

is a 2 -basis for $n$ with $2 \cdot[\sqrt{n}]+1$ elements, thus

$$
\liminf _{n \rightarrow \infty} \frac{n}{k^{2}} \geq \frac{1}{4}
$$

(see Rohrbach [6]).

Mrose [5] proved that $\liminf _{n \rightarrow \infty} n / k^{2} \geq 2 / 7=0.2857 \ldots$

Rohrbach [6] gave a nontrivial upper bound with combinatorial argument: $\lim \sup _{n \rightarrow \infty} n / k^{2} \leq 0.4992$. Moser [3] improved this estimate with analytic argument (0.4903), and later, Moser, Pounder and Riddell [4] showed that $\lim \sup _{n \rightarrow \infty} n / k^{2} \leq 0.4847$. W. Klotz [2] proved that $\lim \sup _{n \rightarrow \infty} n / k^{2}$ $\leq 0.4802$.

Güntürk and Nathanson [1], using Fourier series for functions of two variables, showed that 0.4802 can be replaced by 0.4789 . We will prove the following theorem (using Fourier series for functions of one variable):

Theorem. $\lim \sup _{n \rightarrow \infty} n / k^{2} \leq 0.4778$.

2000 Mathematics Subject Classification: Primary 11B34.

Key words and phrases: additive number theory, general sequences, finite additive bases. 
2. Proof of the Theorem. Let $n$ be a fixed large positive integer and let $F(z)=\sum_{l=1}^{k} z^{a_{l}}$ be the generating function of the sequence $\mathcal{A}$ (where $\mathcal{A}$ is a minimal basis for $n$ ). Then

$$
\frac{1}{2}\left(F^{2}(z)+F\left(z^{2}\right)\right)=1+z+z^{2}+\cdots+z^{n}+\sum_{j=0}^{2 n} \delta(j) z^{j},
$$

where $\delta(j) \geq 0$ for all $j$, because $\mathcal{A}$ is a 2-basis for $n$.

By (1), for $z=1$ we have

$$
\frac{1}{2}\left(k^{2}+k\right)=n+1+\sum_{j=0}^{2 n} \delta(j) .
$$

Similarly to the proof of Moser, we will show that $\sum_{j=0}^{2 n} \delta(j)$ is "large".

Let $z=e\left(\frac{t}{n+1}\right)=e^{2 \pi i t /(n+1)}$, where $t$ is a positive integer. For $(n+1) \nmid t$, we obtain $1+z+z^{2}+\cdots+z^{n}=0$, thus by (1),

$$
\begin{aligned}
& \sum_{j=0}^{2 n} \delta(j) \geq\left|\sum_{j=0}^{2 n} \delta(j) e\left(\frac{j t}{n+1}\right)\right| \\
= & \frac{1}{2}\left|\left(\sum_{l=1}^{k} e\left(\frac{t a_{l}}{n+1}\right)\right)^{2}+\sum_{l=1}^{k} e\left(\frac{2 t a_{l}}{n+1}\right)\right| \geq \frac{1}{2}\left(\left|\sum_{l=1}^{k} e\left(\frac{t a_{l}}{n+1}\right)\right|^{2}-k\right) \\
= & \frac{1}{2}\left(\left(\sum_{l=1}^{k} \cos \frac{2 \pi t a_{l}}{n+1}\right)^{2}+\left(\sum_{l=1}^{k} \sin \frac{2 \pi t a_{l}}{n+1}\right)^{2}\right)-\frac{k}{2} .
\end{aligned}
$$

We shall need the following lemma.

$$
\begin{aligned}
& \text { Lemma. Let } 0<\beta<1,0<\varepsilon \leq(1-\beta) / 2 \text { and } \\
& f(x)= \begin{cases}1 & \text { if } 0 \leq x \leq 2 \pi \beta, \\
1-\frac{x-2 \pi \beta}{2 \pi \varepsilon(1-\beta-\varepsilon)} & \text { if } 2 \pi \beta \leq x \leq 2 \pi(\beta+\varepsilon), \\
1-\frac{1}{1-\beta-\varepsilon} & \text { if } 2 \pi(\beta+\varepsilon) \leq x \leq(1-\varepsilon) 2 \pi, \\
1-\frac{2 \pi-x}{2 \pi \varepsilon(1-\beta-\varepsilon)} & \text { if }(1-\varepsilon) 2 \pi \leq x \leq 2 \pi .\end{cases}
\end{aligned}
$$

Then the Fourier series of $f$ is

$$
\begin{aligned}
& \sum_{t=1}^{\infty}\left(\frac{2}{\pi^{2} \varepsilon(1-\beta-\varepsilon)} \cdot \frac{\sin (\pi t \varepsilon) \sin (\pi t(\beta+\varepsilon)) \cos (\pi t \beta)}{t^{2}} \cos (t x)\right. \\
& \left.\quad+\frac{2}{\pi^{2} \varepsilon(1-\beta-\varepsilon)} \cdot \frac{\sin (\pi t \varepsilon) \sin (\pi t(\beta+\varepsilon)) \sin (\pi t \beta)}{t^{2}} \sin (t x)\right) .
\end{aligned}
$$


Proof. Let $0<d \leq 1 / 2$ and

$$
\varrho_{d}(x)= \begin{cases}1-|x| / d 2 \pi & \text { if }|x| \leq d 2 \pi \\ 0 & \text { if } d 2 \pi \leq|x| \leq \pi\end{cases}
$$

Then

$$
\begin{aligned}
f(x)=1 & -\frac{1-\beta}{2 \varepsilon(1-\beta-\varepsilon)} \varrho_{(1-\beta) / 2}(x-(1+\beta) \pi) \\
& +\frac{1}{1-\beta-\varepsilon} \cdot \frac{1-\beta-2 \varepsilon}{2 \varepsilon} \varrho_{(1-\beta-2 \varepsilon) / 2}(x-(1+\beta) \pi) .
\end{aligned}
$$

If we denote the Fourier series of the function $\varrho_{d}(x)$ by

$$
u_{0}+\sum_{t=1}^{\infty} u_{t} \cos (t x)+\sum_{t=1}^{\infty} v_{t} \sin (t x)
$$

then

$$
u_{0}=\frac{1}{2 \pi} \int_{-\pi}^{\pi} \varrho_{d}(x) d x=d
$$

and for $t>0$,

$$
\begin{aligned}
& u_{t}=\frac{1}{\pi} \int_{-\pi}^{\pi} \varrho_{d}(x) \cos (-t x) d x, \\
& v_{t}=-\frac{1}{\pi} \int_{-\pi}^{\pi} \varrho_{d}(x) \sin (-t x) d x=0 .
\end{aligned}
$$

By (7),

$$
\begin{aligned}
u_{t} & =\frac{2}{\pi} \int_{0}^{d 2 \pi}\left(1-\frac{1}{d 2 \pi} x\right) \cos (-t x) d x \\
& =\frac{2}{\pi}\left[\frac{\sin (-t x)}{-t}\left(1-\frac{1}{d 2 \pi} x\right)-\frac{\cos (-t x)}{t^{2} d 2 \pi}\right]_{0}^{d 2 \pi} \\
& =\frac{2}{\pi}\left(\frac{-\cos (-t d 2 \pi)}{t^{2} d 2 \pi}+\frac{1}{t^{2} d 2 \pi}\right)=\frac{1-\cos (t d 2 \pi)}{t^{2} d \pi^{2}} .
\end{aligned}
$$

Therefore by (5), (6) and (8), we have

$$
\varrho_{d}(x)=d+\sum_{t=1}^{\infty} \frac{1-\cos (t d 2 \pi)}{t^{2} d \pi^{2}} \cos (t x),
$$


hence in view of (4),

$$
\begin{aligned}
f(x)= & 1-\frac{1-\beta}{2 \varepsilon(1-\beta-\varepsilon)}\left(\frac{1-\beta}{2}+\sum_{t=1}^{\infty} \frac{1-\cos (t(1-\beta) \pi)}{t^{2} \frac{1-\beta}{2} \pi^{2}}\right. \\
& \times \cos (t(x-(1+\beta) \pi)))+\frac{1}{1-\beta-\varepsilon} \cdot \frac{1-\beta-2 \varepsilon}{2 \varepsilon}\left(\frac{1-\beta-2 \varepsilon}{2}\right. \\
& \left.+\sum_{t=1}^{\infty} \frac{1-\cos (t(1-\beta-2 \varepsilon) \pi)}{t^{2} \frac{1-\beta-2 \varepsilon}{2} \pi^{2}} \cos (t(x-(1+\beta) \pi))\right) \\
= & 1-\frac{(1-\beta)^{2}}{4 \varepsilon(1-\beta-\varepsilon)}+\frac{(1-\beta-2 \varepsilon)^{2}}{4 \varepsilon(1-\beta-\varepsilon)} \\
& +\sum_{t=1}^{\infty} \frac{1}{\varepsilon(1-\beta-\varepsilon) \pi^{2} t^{2}}(\cos (t(1-\beta) \pi)-\cos (t(1-\beta-2 \varepsilon) \pi)) \\
& \times \cos (t(x-(1+\beta) \pi)) \\
= & \sum_{t=1}^{\infty} \frac{2 \sin (t(1-\beta-\varepsilon) \pi) \sin (-t \varepsilon \pi)}{\varepsilon(1-\beta-\varepsilon) \pi^{2} t^{2}} \\
& \times(\cos (t x) \cos (t(1+\beta) \pi)+\sin (t x) \sin (t(1+\beta) \pi)) \\
= & \sum_{t=1}^{\infty} \frac{2 \sin (\pi t(\beta+\varepsilon)) \sin (\pi t \varepsilon)}{\varepsilon(1-\beta-\varepsilon) \pi^{2} t^{2}}(\cos (t x) \cos (\pi t \beta)+\sin (t x) \sin (\pi t \beta)),
\end{aligned}
$$

which completes the proof of the lemma.

Let $A(y)=\left|\left\{a_{l} \in \mathcal{A} \mid a_{l} \leq y\right\}\right|$. Then by the lemma,

$$
\begin{aligned}
A(\beta n)-\frac{\beta+\varepsilon}{1-\beta-\varepsilon}(k-A(\beta n)) \leq \sum_{l=1}^{k} f\left(\frac{2 \pi a_{l}}{n+1}\right) \\
=\sum_{l=1}^{k} \frac{2}{\pi^{2} \varepsilon(1-\beta-\varepsilon)} \sum_{t=1}^{\infty}\left(\frac{\sin (\pi t \varepsilon) \sin (\pi t(\beta+\varepsilon))}{t^{2}}\right. \\
\left.\quad \times\left(\cos (\pi t \beta) \cos \frac{2 \pi t a_{l}}{n+1}+\sin (\pi t \beta) \sin \frac{2 \pi t a_{l}}{n+1}\right)\right) \\
=\frac{2}{\pi^{2} \varepsilon(1-\beta-\varepsilon)} \sum_{t=1}^{\infty}\left(\frac{\sin (\pi t \varepsilon) \sin (\pi t(\beta+\varepsilon))}{t^{2}}\right. \\
\left.\quad \times\left(\cos (\pi t \beta) \sum_{l=1}^{k} \cos \frac{2 \pi t a_{l}}{n+1}+\sin (\pi t \beta) \sum_{l=1}^{k} \sin \frac{2 \pi t a_{l}}{n+1}\right)\right)
\end{aligned}
$$




$$
\begin{aligned}
\leq & \frac{2}{\pi^{2} \varepsilon(1-\beta-\varepsilon)} \sum_{\substack{t=1 \\
(n+1) \nmid t}}^{\infty} \frac{|\sin (\pi t \varepsilon) \sin (\pi t(\beta+\varepsilon))|}{t^{2}} \sqrt{\cos ^{2}(\pi t \beta)+\sin ^{2}(\pi t \beta)} \\
& \times \sqrt{\left(\sum_{l=1}^{k} \cos \frac{2 \pi t a_{l}}{n+1}\right)^{2}+\left(\sum_{l=1}^{k} \sin \frac{2 \pi t a_{l}}{n+1}\right)^{2}}+\frac{2}{\pi^{2} \varepsilon(1-\beta-\varepsilon)} \\
& \times \sum_{s=1}^{\infty} \frac{|\sin (\pi s(n+1) \varepsilon) \sin (\pi s(n+1)(\beta+\varepsilon))|}{s^{2}(n+1)^{2}}|\cos (\pi s(n+1) \beta)| k .
\end{aligned}
$$

It follows from (3) and (9) that

$$
\begin{aligned}
& \frac{1}{1-\beta-\varepsilon}(A(\beta n)-(\beta+\varepsilon) k) \\
& \leq \frac{2}{\pi^{2} \varepsilon(1-\beta-\varepsilon)} \sum_{\substack{t=1 \\
(n+1) \nmid t}}^{\infty} \frac{|\sin (\pi t \varepsilon) \sin (\pi t(\beta+\varepsilon))|}{t^{2}} \sqrt{k+2 \sum_{j=0}^{2 n} \delta(j)} \\
& \quad+\frac{2}{\pi^{2} \varepsilon(1-\beta-\varepsilon)} \cdot \frac{k}{(n+1)^{2}} \sum_{s=1}^{\infty} \frac{1}{s^{2}},
\end{aligned}
$$

which implies that

$$
\begin{aligned}
& A(\beta n) \leq(\beta+\varepsilon) k \\
+ & \frac{1}{\pi^{2} \varepsilon}\left(\sum_{t=1}^{\infty} \frac{|2 \sin (\pi t \varepsilon) \sin (\pi t(\beta+\varepsilon))|}{t^{2}}\right) \sqrt{k+2 \sum_{j=0}^{2 n} \delta(j)+\frac{1}{3 \varepsilon} \cdot \frac{k}{(n+1)^{2}} .}
\end{aligned}
$$

Let

$$
S(\beta, \varepsilon)=\sum_{t=1}^{\infty} \frac{|2 \sin (\pi t \varepsilon) \sin (\pi t(\beta+\varepsilon))|}{t^{2}}
$$

and $0<\tau<1 / 2$ (the value of $\tau$ will be chosen later). If $\sum_{j=0}^{2 n} \delta(j) \geq \tau k^{2}$, then by (2),

$$
n+1 \leq\left(\frac{1}{2}-\tau\right) k^{2}+\frac{1}{2} k .
$$

If $\sum_{j=0}^{2 n} \delta(j) \leq \tau k^{2}$, then by $(10)$,

$$
A(\beta n) \leq(\beta+\varepsilon) k+\frac{1}{\pi^{2} \varepsilon} S(\beta, \varepsilon) \sqrt{\frac{1}{k}+2 \tau} \cdot k+\frac{1}{3 \varepsilon} \cdot \frac{1}{(n+1)^{2}} k .
$$

Let $0<\mu<1 / 2$. Since $a_{l}>n / 2, a_{m}>n / 2$ implies that $a_{l}+a_{m}>n$, and also $(1 / 2-\mu) n<a_{l} \leq n / 2,(1 / 2+\mu) n<a_{m} \leq n$ implies that $a_{l}+a_{m}>n$, 
we have

$$
\begin{aligned}
n+1 \leq & \frac{k^{2}+k}{2}-\frac{(k-A(n / 2))(k-A(n / 2)+1)}{2} \\
& -\left(A\left(\frac{n}{2}\right)-A\left(\left(\frac{1}{2}-\mu\right) n\right)\right)\left(k-A\left(\left(\frac{1}{2}+\mu\right) n\right)\right) \\
\leq & \frac{1}{2} k^{2}+\frac{1}{2} k-\frac{1}{2}\left(k-A\left(\frac{n}{2}\right)\right)^{2} \\
& -\left(A\left(\frac{n}{2}\right)-A\left(\left(\frac{1}{2}-\mu\right) n\right)\right)\left(k-A\left(\left(\frac{1}{2}+\mu\right) n\right)\right),
\end{aligned}
$$

which can be written as

$$
\begin{aligned}
& -\frac{1}{2}\left(A\left(\frac{n}{2}\right)\right)^{2}+A\left(\left(\frac{1}{2}+\mu\right) n\right) A\left(\frac{n}{2}\right)+\frac{1}{2} k \\
& +A\left(\left(\frac{1}{2}-\mu\right) n\right)\left(k-A\left(\left(\frac{1}{2}+\mu\right) n\right)\right) \\
& =-\frac{1}{2}\left(A\left(\frac{n}{2}\right)-A\left(\left(\frac{1}{2}+\mu\right) n\right)\right)^{2}+\frac{1}{2}\left(A\left(\left(\frac{1}{2}+\mu\right) n\right)\right)^{2}+\frac{1}{2} k \\
& +A\left(\left(\frac{1}{2}-\mu\right) n\right)\left(k-A\left(\left(\frac{1}{2}+\mu\right) n\right)\right) .
\end{aligned}
$$

By $(13)$, for $\beta=1 / 2-\mu$ and $\varepsilon=\varepsilon_{1}\left(0<\varepsilon_{1} \leq 1 / 4+\mu / 2\right)$ we have

$$
\begin{aligned}
A\left(\left(\frac{1}{2}-\mu\right) n\right) & \leq\left(\frac{1}{2}-\mu+\varepsilon_{1}\right) k \\
& +\frac{1}{\pi^{2} \varepsilon_{1}} S\left(\frac{1}{2}-\mu, \varepsilon_{1}\right) \sqrt{\frac{1}{k}+2 \tau} \cdot k+\frac{1}{3 \varepsilon_{1}} \cdot \frac{1}{(n+1)^{2}} k .
\end{aligned}
$$

For $\beta=1 / 2+\mu$ and $\varepsilon=\varepsilon_{2}\left(0<\varepsilon_{2}<1 / 4-\mu / 2\right)$, by $(13)$,

$$
\begin{aligned}
A\left(\left(\frac{1}{2}+\mu\right) n\right) & \leq\left(\frac{1}{2}+\mu+\varepsilon_{2}\right) k \\
& +\frac{1}{\pi^{2} \varepsilon_{2}} S\left(\frac{1}{2}+\mu, \varepsilon_{2}\right) \sqrt{\frac{1}{k}+2 \tau} \cdot k+\frac{1}{3 \varepsilon_{2}} \cdot \frac{1}{(n+1)^{2}} k .
\end{aligned}
$$

From (13), for $\beta=1 / 2$ and $\varepsilon=\varepsilon_{0}\left(0<\varepsilon_{0}<1 / 4\right)$ we get

$$
\begin{aligned}
A\left(\frac{n}{2}\right) \leq & \left(\frac{1}{2}+\varepsilon_{0}\right) k \\
& +\frac{1}{\pi^{2} \varepsilon_{0}} S\left(\frac{1}{2}, \varepsilon_{0}\right) \sqrt{\frac{1}{k}+2 \tau} \cdot k+\frac{1}{3 \varepsilon_{0}} \cdot \frac{1}{(n+1)^{2}} k .
\end{aligned}
$$

We will distinguish two cases. If the right-hand side of (18) is not greater than $A((1 / 2+\mu) n)$ (the first case), then replacing in $(15) A(n / 2)$ by the right-hand side of (18), by (14), we obtain 


$$
\begin{aligned}
n+1 \leq & \frac{1}{2} k^{2}+\frac{1}{2} k \\
& -\frac{1}{2}\left(\frac{1}{2}-\varepsilon_{0}-\frac{1}{\pi^{2} \varepsilon_{0}} S\left(\frac{1}{2}, \varepsilon_{0}\right) \sqrt{\frac{1}{k}+2 \tau}-\frac{1}{3 \varepsilon_{0}} \cdot \frac{1}{(n+1)^{2}}\right)^{2} k^{2} \\
& -\left(\left(\frac{1}{2}+\varepsilon_{0}+\frac{1}{\pi^{2} \varepsilon_{0}} S\left(\frac{1}{2}, \varepsilon_{0}\right) \sqrt{\frac{1}{k}+2 \tau}+\frac{1}{3 \varepsilon_{0}} \cdot \frac{1}{(n+1)^{2}}\right) k\right. \\
& \left.-A\left(\left(\frac{1}{2}-\mu\right) n\right)\right)\left(k-A\left(\left(\frac{1}{2}+\mu\right) n\right)\right) .
\end{aligned}
$$

Hence in view of (16) and (17),

$$
\begin{aligned}
& n+1 \leq \frac{1}{2} k^{2}+\frac{1}{2} k \\
& -\frac{1}{2}\left(\frac{1}{2}-\varepsilon_{0}-\frac{1}{\pi^{2} \varepsilon_{0}} S\left(\frac{1}{2}, \varepsilon_{0}\right) \sqrt{\frac{1}{k}+2 \tau}-\frac{1}{3 \varepsilon_{0}} \cdot \frac{1}{(n+1)^{2}}\right)^{2} k^{2} \\
& -\left(\mu+\varepsilon_{0}-\varepsilon_{1}+\frac{1}{\pi^{2}} \sqrt{\frac{1}{k}+2 \tau}\left(\frac{S\left(1 / 2, \varepsilon_{0}\right)}{\varepsilon_{0}}-\frac{S\left(1 / 2-\mu, \varepsilon_{1}\right)}{\varepsilon_{1}}\right)\right. \\
& \left.+\left(\frac{1}{3 \varepsilon_{0}}-\frac{1}{3 \varepsilon_{1}}\right) \cdot \frac{1}{(n+1)^{2}}\right) \\
& \quad \times\left(\frac{1}{2}-\mu-\varepsilon_{2}-\frac{1}{\pi^{2} \varepsilon_{2}} S\left(\frac{1}{2}+\mu, \varepsilon_{2}\right) \sqrt{\frac{1}{k}+2 \tau}-\frac{1}{3 \varepsilon_{2}} \cdot \frac{1}{(n+1)^{2}}\right) k^{2}
\end{aligned}
$$

(if the right-hand side of (17) is less than or equal to $k$ ).

If the right-hand side of (18) is greater than $A((1 / 2+\mu) n)$ (the second case), then we may suppose that $k-A(n / 2) \leq \sqrt{2 \tau} \cdot k$, i.e.,

$$
A\left(\frac{n}{2}\right) \geq(1-\sqrt{2 \tau}) k,
$$

otherwise (14) would imply $n+1 \leq \frac{1}{2} k^{2}+\frac{1}{2} k-\frac{1}{2}(k-A(n / 2))^{2} \leq$ $(1 / 2-\tau) k^{2}+\frac{1}{2} k$, which is identical with (12). So by (14), (18) and (16),

$$
\text { (21) } \begin{aligned}
n+1 \leq & \frac{1}{2} k^{2}+\frac{1}{2} k \\
- & \frac{1}{2}\left(\frac{1}{2}-\varepsilon_{0}-\frac{1}{\pi^{2} \varepsilon_{0}} S\left(\frac{1}{2}, \varepsilon_{0}\right) \sqrt{\frac{1}{k}+2 \tau}-\frac{1}{3 \varepsilon_{0}} \cdot \frac{1}{(n+1)^{2}}\right)^{2} k^{2} \\
-\left(\frac{1}{2}-\sqrt{2} \cdot \sqrt{\tau}\right. & \left.+\mu-\varepsilon_{1}-\frac{1}{\pi^{2} \varepsilon_{1}} S\left(\frac{1}{2}-\mu, \varepsilon_{1}\right) \sqrt{\frac{1}{k}+2 \tau}-\frac{1}{3 \varepsilon_{1}} \cdot \frac{1}{(n+1)^{2}}\right) \\
& \times\left(\frac{1}{2}-\varepsilon_{0}-\frac{1}{\pi^{2} \varepsilon_{0}} S\left(\frac{1}{2}, \varepsilon_{0}\right) \sqrt{\frac{1}{k}+2 \tau}-\frac{1}{3 \varepsilon_{0}} \cdot \frac{1}{(n+1)^{2}}\right) k^{2}
\end{aligned}
$$

(provided that the right-hand side of (18) is less than or equal to $k$ ). 
Thus we have one of the estimates (12), (19) and (21). Let $\mu=\varepsilon_{1}=\varepsilon_{2}=$ $1 / 12, \varepsilon_{0}=1 / 14$ and $\sqrt{\tau}=0.149$, i.e., $\tau=0.022201$. Then $1 / 2-\tau<0.4778$, so (12) implies the theorem.

By (11),

$$
\begin{aligned}
S\left(\frac{5}{12}, \frac{1}{12}\right)= & \sum_{t=1}^{\infty} \frac{2\left|\sin \frac{\pi t}{12}\right| \cdot\left|\sin \frac{\pi t}{2}\right|}{t^{2}} \\
= & 2\left(\sin \frac{\pi}{12} \sum_{s=0}^{\infty}\left(\frac{1}{(12 s+1)^{2}}+\frac{1}{(12 s+11)^{2}}\right)\right. \\
& +\frac{\sqrt{2}}{2} \sum_{s=0}^{\infty}\left(\frac{1}{(12 s+3)^{2}}+\frac{1}{(12 s+9)^{2}}\right) \\
& \left.+\sin \frac{5 \pi}{12} \sum_{s=0}^{\infty}\left(\frac{1}{(12 s+5)^{2}}+\frac{1}{(12 s+7)^{2}}\right)\right)
\end{aligned}
$$

where

$$
\begin{aligned}
\frac{\sqrt{2}}{2} \sum_{s=0}^{\infty}\left(\frac{1}{(12 s+3)^{2}}+\right. & \left.\frac{1}{(12 s+9)^{2}}\right) \\
& =\frac{\sqrt{2}}{2}\left(\sum_{s=1}^{\infty} \frac{1}{(3 s)^{2}}-\sum_{s=1}^{\infty} \frac{1}{(6 s)^{2}}\right) \\
& =\frac{\sqrt{2}}{2} \cdot \frac{\pi^{2}}{6}\left(\frac{1}{9}-\frac{1}{36}\right)=\frac{\sqrt{2} \pi^{2}}{144}<0.0969287
\end{aligned}
$$

Since $\sum_{s=1}^{m} \frac{1}{s^{2}}>\frac{m(2 m-1)}{3(2 m+1)^{2}} \pi^{2}$, it follows that

$$
\begin{aligned}
\sum_{s=0}^{\infty} & \left(\frac{1}{(12 s+1)^{2}}+\frac{1}{(12 s+11)^{2}}\right) \\
& \leq \sum_{s=0}^{M}\left(\frac{1}{(12 s+1)^{2}}+\frac{1}{(12 s+11)^{2}}\right)+\sum_{s=M+1}^{\infty}\left(\frac{1}{(12 s)^{2}}+\frac{1}{(12 s+6)^{2}}\right) \\
& =\sum_{s=0}^{M}\left(\frac{1}{(12 s+1)^{2}}+\frac{1}{(12 s+11)^{2}}\right)+\frac{1}{36}\left(\frac{\pi^{2}}{6}-\sum_{s=1}^{2 M+1} \frac{1}{s^{2}}\right) \\
& \leq \sum_{s=0}^{M}\left(\frac{1}{(12 s+1)^{2}}+\frac{1}{(12 s+11)^{2}}\right)+\frac{1}{36}\left(\frac{\pi^{2}}{6}-\frac{(2 M+1)(4 M+1)}{3(4 M+3)^{2}} \pi^{2}\right) \\
& =\sum_{s=0}^{M}\left(\frac{1}{(12 s+1)^{2}}+\frac{1}{(12 s+11)^{2}}\right)+\frac{\pi^{2}}{216} \cdot \frac{12 M+7}{(4 M+3)^{2}}
\end{aligned}
$$


and (by aid of computer) we find that for $M=79$ this is less than 1.02342 . Similarly,

$$
\begin{aligned}
\sum_{s=0}^{\infty}\left(\frac{1}{(12 s+5)^{2}}\right. & \left.+\frac{1}{(12 s+7)^{2}}\right) \\
& \leq \sum_{s=0}^{M}\left(\frac{1}{(12 s+5)^{2}}+\frac{1}{(12 s+7)^{2}}\right)+\frac{\pi^{2}}{216} \cdot \frac{12 M+7}{(4 M+3)^{2}}
\end{aligned}
$$

and for $M=84$, the right-hand side of this inequality is less than 0.0737 . Furthermore, $\sin \frac{\pi}{12}<0.25881905$ and $\sin \frac{5 \pi}{12}<0.96592583$, by (22) and (23) we have

(24) $\quad S\left(\frac{5}{12}, \frac{1}{12}\right)$

$\leq 2(0.25881905 \cdot 1.02342+0.0969287+0.96592583 \cdot 0.0737)<0.866$.

By (11),

$$
\begin{aligned}
S\left(\frac{7}{12}, \frac{1}{12}\right)= & \sum_{t=1}^{\infty} \frac{2\left|\sin \frac{\pi t}{12}\right| \cdot\left|\sin \frac{2 \pi t}{3}\right|}{t^{2}} \\
= & \sqrt{3}\left(\sin \frac{\pi}{12} \sum_{s=0}^{\infty}\left(\frac{1}{(12 s+1)^{2}}+\frac{1}{(12 s+11)^{2}}\right)\right. \\
& +\frac{1}{2} \sum_{s=0}^{\infty}\left(\frac{1}{(12 s+2)^{2}}+\frac{1}{(12 s+10)^{2}}\right) \\
& +\frac{\sqrt{3}}{2} \sum_{s=0}^{\infty}\left(\frac{1}{(12 s+4)^{2}}+\frac{1}{(12 s+8)^{2}}\right) \\
& \left.+\sin \frac{5 \pi}{12} \sum_{s=0}^{\infty}\left(\frac{1}{(12 s+5)^{2}}+\frac{1}{(12 s+7)^{2}}\right)\right),
\end{aligned}
$$

where

$$
\sum_{s=0}^{\infty}\left(\frac{1}{(12 s+4)^{2}}+\frac{1}{(12 s+8)^{2}}\right)=\frac{1}{16} \cdot \frac{\pi^{2}}{6}\left(1-\frac{1}{9}\right)=\frac{\pi^{2}}{108}<0.0913853
$$

and

$$
\begin{aligned}
& \sum_{s=0}^{\infty}\left(\frac{1}{(12 s+2)^{2}}+\frac{1}{(12 s+10)^{2}}\right) \\
& \quad \leq \sum_{s=0}^{83}\left(\frac{1}{(12 s+2)^{2}}+\frac{1}{(12 s+10)^{2}}\right)+\frac{\pi^{2}}{216} \cdot \frac{12 \cdot 83+7}{(4 \cdot 83+3)^{2}}<0.2744
\end{aligned}
$$


Hence

$$
\begin{aligned}
S\left(\frac{7}{12}, \frac{1}{12}\right) \leq & \sqrt{3}\left(0.25881905 \cdot 1.02342+\frac{1}{2} \cdot 0.2744\right. \\
& \left.+\frac{\sqrt{3}}{2} \cdot 0.0913853+0.96592583 \cdot 0.0737\right)<0.95681
\end{aligned}
$$

Again by (11),

$$
\begin{aligned}
S\left(\frac{1}{2}, \frac{1}{14}\right)= & \sum_{t=1}^{\infty} \frac{2\left|\sin \frac{\pi t}{14}\right| \cdot\left|\sin \frac{4 \pi t}{7}\right|}{t^{2}} \\
= & 2 \sin \frac{\pi}{14} \sin \frac{4 \pi}{7} \sum_{s=0}^{\infty}\left(\frac{1}{(14 s+1)^{2}}+\frac{1}{(14 s+13)^{2}}\right) \\
& +2\left(\sin \frac{\pi}{7}\right)^{2} \sum_{s=0}^{\infty}\left(\frac{1}{(14 s+2)^{2}}+\frac{1}{(14 s+12)^{2}}\right) \\
& +2 \sin \frac{3 \pi}{14} \sin \frac{2 \pi}{7} \sum_{s=0}^{\infty}\left(\frac{1}{(14 s+3)^{2}}+\frac{1}{(14 s+11)^{2}}\right) \\
& +2\left(\sin \frac{2 \pi}{7}\right)^{2} \sum_{s=0}^{\infty}\left(\frac{1}{(14 s+4)^{2}}+\frac{1}{(14 s+10)^{2}}\right) \\
& +2 \sin \frac{5 \pi}{14} \sin \frac{\pi}{7} \sum_{s=0}^{\infty}\left(\frac{1}{(14 s+5)^{2}}+\frac{1}{(14 s+9)^{2}}\right) \\
& +2\left(\sin \frac{3 \pi}{7}\right)^{2} \sum_{s=0}^{\infty}\left(\frac{1}{(14 s+6)^{2}}+\frac{1}{(14 s+8)^{2}}\right)
\end{aligned}
$$

where

$$
\begin{aligned}
& \sum_{s=0}^{\infty}\left(\frac{1}{(14 s+1)^{2}}+\frac{1}{(14 s+13)^{2}}\right) \\
& \quad \leq \sum_{s=0}^{M}\left(\frac{1}{(14 s+1)^{2}}+\frac{1}{(14 s+13)^{2}}\right)+\sum_{s=M+1}^{\infty}\left(\frac{1}{(14 s)^{2}}+\frac{1}{(14 s+7)^{2}}\right) \\
& \quad \leq \sum_{s=0}^{M}\left(\frac{1}{(14 s+1)^{2}}+\frac{1}{(14 s+13)^{2}}\right)+\frac{1}{49}\left(\frac{\pi^{2}}{6}-\frac{(2 M+1)(4 M+1)}{3(4 M+3)^{2}} \pi^{2}\right) \\
& \quad=\sum_{s=0}^{M}\left(\frac{1}{(14 s+1)^{2}}+\frac{1}{(14 s+13)^{2}}\right)+\frac{\pi^{2}}{294} \cdot \frac{12 M+7}{(4 M+3)^{2}},
\end{aligned}
$$

which is less than 1.0171 (let $M=103$ ). Similarly, setting $M=90, M=90$, $M=87, M=89$ and $M=92$ in the estimates of the other series of the right-hand side of (26), respectively, we get 
(27) $S\left(\frac{1}{2}, \frac{1}{14}\right) \leq 0.4338837392 \cdot 1.0171+0.3765101982 \cdot 0.26765$

$+0.9749279122 \cdot 0.1297+1.222520934 \cdot 0.08255$

$+0.7818314825 \cdot 0.0622+1.900968868 \cdot 0.05314$ $<0.9191$.

On the other hand, by (26),

$$
\begin{aligned}
S\left(\frac{1}{2}, \frac{1}{14}\right) \geq & 2 \sin \frac{\pi}{14} \sin \frac{4 \pi}{7} \sum_{s=0}^{1}\left(\frac{1}{(14 s+1)^{2}}+\frac{1}{(14 s+13)^{2}}\right) \\
& +2\left(\sin \frac{\pi}{7}\right)^{2} \sum_{s=0}^{1}\left(\frac{1}{(14 s+2)^{2}}+\frac{1}{(14 s+12)^{2}}\right) \\
& +2 \sin \frac{3 \pi}{14} \sin \frac{2 \pi}{7} \sum_{s=0}^{2}\left(\frac{1}{(14 s+3)^{2}}+\frac{1}{(14 s+11)^{2}}\right) \\
& +2\left(\sin \frac{2 \pi}{7}\right)^{2} \sum_{s=0}^{4}\left(\frac{1}{(14 s+4)^{2}}+\frac{1}{(14 s+10)^{2}}\right) \\
& +2 \sin \frac{5 \pi}{14} \sin \frac{\pi}{7} \sum_{s=0}^{5}\left(\frac{1}{(14 s+5)^{2}}+\frac{1}{(14 s+9)^{2}}\right) \\
& +2\left(\sin \frac{3 \pi}{7}\right)^{2} \sum_{s=0}^{3}\left(\frac{1}{(14 s+6)^{2}}+\frac{1}{(14 s+8)^{2}}\right) \\
\geq & 0.43388 \cdot 1.01+0.37651 \cdot 0.26+0.97492 \cdot 0.125 \\
& +1.22252 \cdot 0.08+0.78183 \cdot 0.06+1.90096 \cdot 0.05 \\
& 0.8976 .
\end{aligned}
$$

Now, by (19), (27), (28), (24) and (25), for sufficiently large $n(k(n) \rightarrow \infty$ as $n \rightarrow \infty)$ we obtain

$$
\begin{aligned}
n+1 \leq \frac{1}{2} k^{2}+ & \frac{1}{2} k \\
- & \frac{1}{2}\left(\frac{3}{7}-\frac{14}{\pi^{2}} \cdot 0.9191 \cdot \sqrt{\frac{1}{k}+2 \cdot 0.022201}-\frac{14}{3} \cdot \frac{1}{(n+1)^{2}}\right)^{2} k^{2} \\
-\left(\frac{1}{14}+\frac{1}{\pi^{2}}\right. & \left.\cdot \sqrt{\frac{1}{k}+2 \cdot 0.022201}(14 \cdot 0.8976-12 \cdot 0.866)+\frac{2}{3} \cdot \frac{1}{(n+1)^{2}}\right) \\
& \times\left(\frac{1}{3}-\frac{12}{\pi^{2}} \cdot 0.95681 \cdot \sqrt{\frac{1}{k}+2 \cdot 0.022201}-4 \cdot \frac{1}{(n+1)^{2}}\right) k^{2} \\
\leq & \frac{1}{2} k^{2}+\frac{1}{2} k-\frac{1}{2}(0.153845)^{2} k^{2}-0.11785 \cdot 0.08819 \cdot k^{2}<0.4778 k^{2},
\end{aligned}
$$

thus (19) implies the theorem. 


$$
\begin{aligned}
& \text { By }(21),(27) \text { and }(24), \\
& n+1 \leq \frac{1}{2} k^{2}+\frac{1}{2} k \\
&-\frac{1}{2}\left(\frac{3}{7}-\frac{14}{\pi^{2}} \cdot 0.9191 \cdot \sqrt{\frac{1}{k}+2 \cdot 0.022201}-\frac{14}{3} \cdot \frac{1}{(n+1)^{2}}\right)^{2} k^{2} \\
&-\left(\frac{1}{2}-\sqrt{2} \cdot 0.149-\frac{12}{\pi^{2}} \cdot 0.866 \cdot \sqrt{\frac{1}{k}+2 \cdot 0.022201}-4 \cdot \frac{1}{(n+1)^{2}}\right) \\
& \times\left(\frac{3}{7}-\frac{14}{\pi^{2}} \cdot 0.9191 \cdot \sqrt{\frac{1}{k}+2 \cdot 0.022201}-\frac{14}{3} \cdot \frac{1}{(n+1)^{2}}\right) k^{2} \\
& \leq \frac{1}{2} k^{2}+\frac{1}{2} k-\frac{1}{2}(0.153845)^{2} k^{2}-0.067407 \cdot 0.153845 \cdot k^{2} \\
&< 0.4778 k^{2},
\end{aligned}
$$

therefore (21) also implies the theorem, which completes the proof.

\section{References}

[1] C. S. Güntürk and M. B. Nathanson, A new upper bound for finite additive bases, Acta Arith. 124 (2006), 235-255.

[2] W. Klotz, Eine obere Schranke für die Reichweite einer Extremalbasis zweiter Ordnung, J. Reine Angew. Math. 238 (1969), 161-168.

[3] L. Moser, On the representation of $1,2, \ldots, n$ by sums, Acta Arith. 6 (1960), 11-13.

[4] L. Moser, J. R. Pounder and J. Riddell, On the cardinality of h-bases for $n$, J. London Math. Soc. 44 (1969), 397-407.

[5] A. Mrose, Untere Schranken für die Reichweiten von Extremalbasen fester Ordnung, Abh. Math. Sem. Univ. Hamburg 48 (1979), 118-124.

[6] H. Rohrbach, Ein Beitrag zur additiven Zahlentheorie, Math. Z. 42 (1936), 1-30.

Department of Mathematical Analysis

Institute of Mathematics

College of Dunaújváros

Táncsics M. u. 1/A.

H-2401, Dunaújváros, Hungary

E-mail: ghorvath@mail.duf.hu

Received on 7.5.2007

and in revised form on 4.9.2007 